\title{
Supernovæ and Transients with EUCLID and the European ELT
}

\author{
Isobel Hook Hon $^{1,2}$ \\ ${ }^{1}$ Oxford Astrophysics, University of Oxford, Oxford, OX1 3RH, U.K. \\ email: imh@astro.ox.ac.uk \\ ${ }^{2}$ INAF-Osservatorio Astronomico di Roma, Monte Porzio Catone, Italy
}

\begin{abstract}
The prospects are described for studies of large samples of supernovæ and other variable objects with two proposed future facilities: (1) the European Extremely Large Telescope, a general-purpose 40-m-class ground-based optical-IR telescope, and (2) EucLID, an M-class mission within ESA's Cosmic Vision programme, primarily for cosmology. The capabilities and status of the two facilities are briefly described. Their suitability for the study of time-varying objects in general, and of supernovæ in particular, is discussed. It is shown that EuCLID has the potential for NIR imaging of a few thousand Type Ia supernovæ to intermediate $\mathrm{z}$, while the E-ELT will be capable of spectroscopic and classification measurements of Type Ia supernovæ to $z=4$.
\end{abstract}

Keywords. Telescopes

\section{The European Extremely Large Telescope}

\subsection{Overview}

The European Extremely Large Telescope (E-ELT) project aims to design and construct a 40-m class optical-IR telescope, which will be the largest such telescope in the world. The project is being led by ESO on behalf of its member states. It has been identified by the ASTRONET process as a top priority of European ground-based astronomy (Bode, Cruz \& Molster 2008).

The E-ELT has a very broad science case. The scientific drivers for the telescope have been developed over a period of many years in collaboration with the project's Science Working Group. The wider astronomical community has also provided input through the Design Reference Science Plan (DRSP, Kissler-Patig, Yoldas \& Liske (2009)) and through a series of ESO and OPTICON-sponsored workshops. The workshops have highlighted synergies with ALMA, JWST, SKA and survey facilities in general.

The key driving themes that have emerged range from extra-solar planets to studies of fundamental constants, dark matter and dark energy. There are many science cases that involve the time domain, some examples of which are listed below:

- Identification of variable sources from other facilities

- Solar system: monitoring weather and volcanic activity

- Exo-planets: radial velocities, direct imaging and transit measurements

- The Galactic Centre: orbits of stars close to the central black hole

- Supernovæ

- Gamma-Ray Bursts

- Expansion of the Universe: measuring the effects of Dark Energy in real time

In addition, if fast detectors capable of sub-minute and sub-second resolution are installed, studies of extreme physics (pulsars, neutron stars, black holes) stellar 
phenomena, GRBs, transits and occultations also become possible (Shearer et al. 2010). Finally, future unknown science programmes that we cannot imagine today, may well turn up the most exciting discoveries.

\subsection{Project Status}

The telescope optical design is a 5-mirror design with a 39-m diameter primary mirror. In April 2010 Cerro Armazones in Chile was adopted as the baseline site. As well as being an excellent site for astronomical observations, Cerro Armazones is about $20 \mathrm{~km}$ from the VLT site at Cerro Paranal, providing significant advantages of joint operations of the two facilities. The instrument plan is under development. Phase A studies for 8 instruments and 2 adaptive optics (AO) systems have been completed and two "first light" instruments have been selected: a diffraction-limited near-IR camera that will be fed by AO, and an integral field spectrograph capable of operating with or without AO. The full instrument suite for the E-ELT will be built up over first decade.

Phase B of the E-ELT project was completed in December 2010, following the successful completion of an external design review. Since then the project has been through a cost and risk reduction exercise, resulting in the diameter of the primary mirror being reduced to $39-\mathrm{m}$ (from $42-\mathrm{m}$, although the precise definition of the diameter also changed). The construction cost is approximately 1 billion Euros, to be shared by the ESO member states. The construction proposal will be presented to ESO Council in December 2011. If approved, construction could start in 2012 , leading to first light early in the next decade.

\section{Overview and Status of EUCLID}

EUCLID is a proposed 1.2-m optical-IR space telescope within ESA's Cosmic Vision 2015-2025 programme. Its primary science goal is precison measurements of cosmological parameters via weak lensing and galaxy clustering techniques. The instrumentation consists of an optical imager and a near-infrared (NIR) imager and spectrograph, all with a field of view of $0.5 \mathrm{deg} .{ }^{2}$ The satellite will be launched by a Soyuz rocket and will operate at the L2 Lagrange point for a 6 -year mission duration.

EuCLID will carry out two main surveys, Wide and Deep. The Wide survey will cover $>15000$ deg. $^{2}$, with imaging in a single broad optical $(\mathrm{R}+\mathrm{I}+\mathrm{Z})$ filter to a depth of $\mathrm{H}(\mathrm{AB})=24.5(10 \sigma$ for a point source), imaging in three near infrared bands $(\mathrm{Y}, \mathrm{J}, \mathrm{H})$ to a depth of $\mathrm{H}(\mathrm{AB})=24(5 \sigma$, extended source $)$ and NIR slitless spectroscopy to a depth of $3 \times 10^{-16} \mathrm{~cm}^{-2} \mathrm{~s}^{-1}(3.5 \sigma$ unresolved line flux $)$. EuCLID also has a potential extension to carry out a Galactic-plane survey.

The Deep survey will cover $>40$ deg. ${ }^{2}$, reaching 2 mags deeper than the Wide survey in both optical and NIR imaging and NIR Spectroscopy. The Deep field is primarily for calibration purposes but will enable a vast range of additional science. The depth will be built up by 40 repeat visits, each of which will reach the same depth as the Wide survey. The exact strategy and timing of these observations has yet to be optimised. In the context of this Symposium, the repeat visits to the Deep field are particularly interesting in that they will facilitate the detection of variable and transient objects.

EuCLID has been proposed as an M-class mission, with the Definition Study report ("Red book") submitted in July 2011. ESA's downselection process in October 2011 resulted in selection of EUCLID for the second M-class launch slot. The mission is now working towards the important milestone of its adoption by ESA in June 2012. Launch would then take place in 2019. 


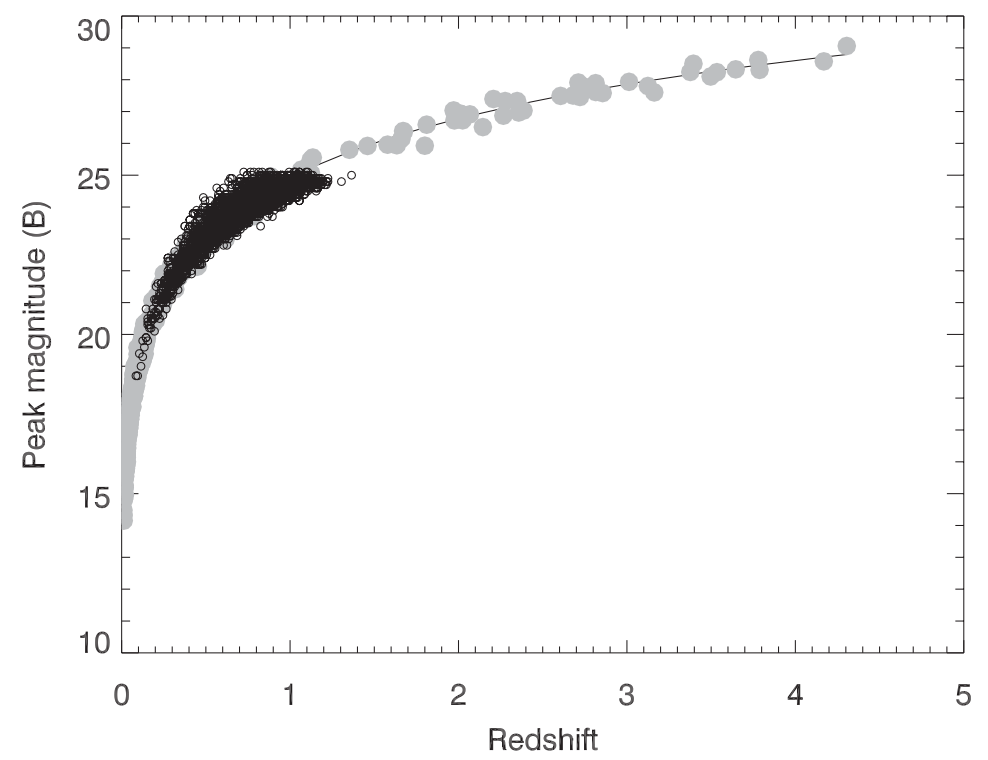

Figure 1. Projected Hubble diagram for Type Ia supernovæ including simulated data from E-ELT and EUCLID. The grey circles with $z<1$ represent the sample that already exists, or will exist within the next few years. These are almost entirely optical observations. The potential sample of SNe with NIR measurements from EuCLID are shown as black dots (simulated data provided by K. Maguire). The circles with $z>1$ represent the sample that could be obtained with a combination of JWST imaging and E-ELT spectroscopic follow-up.

\section{Example of Science Case: Type Ia Supernovæ}

One of the biggest problems facing modern physics is the lack of understanding of Dark Energy which appears to be causing the acceleration of the Universe. Measurements from different techniques including Type Ia supernovæ (SNe Ia), galaxy clustering and the Cosmic Microwave Background are consistent with dark energy being equivalent to the cosmological constant (see, for example, Sullivan et al. 2011), and yet there is no natural explanation for the size of the measured value of its energy density.

SNe Ia are one of the best-known distance indicators; their high luminosity enables them to be used to measure the geometry of the Universe to cosmological distances. Currently there are over 500 well-measured SNe with $z<1$. The main limiting factors in SN cosmology are now the quantity and quality of the nearby SN Ia sample and the related issues of calibration (partially caused by the fact that the nearby and distant SN sets have traditionally been measured through different filter sets) and extinction by dust (in particular disentangling this effect from intrinsic variations in SN luminosity and colour).

Improvements in the nearby sample are now being made by searches such as the Palomar Transient Factory, Skymapper and PanSTARRS. The question of dust extinction and intrinsic luminosity/colour variations is being, or will be, addressed by observing at longer wavelengths where the effects of dust are less pronounced. In total about 80 distant SNe have been measured in the NIR (Freedman et al. 2009, Nobili et al. 2009, Riess et al. 2000). In the next few years we can expect of the order of 100 more to be measured by VISTA combined with optical data from the Dark Energy Survey.

EUCLID has the potential to make more than an order of magnitude improvement in the statistics of SNeIa measured in the near-IR, up to $z \sim 1$, provided a suitable survey strategy is implemented for the repeat visits of the Deep field. Simulations have been 
carried out in order to estimate the number and redshift distribution of SNe observed in the EuCLID deep field. One example is shown in Fig. 1, in which the Deep survey of 6 months total time is divided into periods with $\sim 4$-day and $\sim 10$-day cadences. In this example EuCLID could measure good NIR light curves (at least 4 points per curve) and colours of about 2800 Type Ia SNe to $z \sim 1.2$, of which the $\sim 1700$ with $z<0.8$ would form an unbiased sample for cosmology (Fig.1). We stress that this is just one example, and that the final strategy for the deep field survey has yet to be defined.

At even higher redshifts $(z>2)$ the SNIa Hubble diagram is completely unmeasured. A basic check of the behaviour of the expansion history of the Universe in the matterdominated regime could be made by observing SNeIa at higher redshifts than is currently possible. Such SNe would be within reach of discovery by JWST (again, assuming sufficient time were dedicated to a search). SNe are point sources and so would benefit from the AO correction and the full spatial resolution of the E-ELT. Simulations show that the E-ELT would be capable of obtaining spectra of SNeIa out to $z=4$ (Hook (2010)), using an AO-assisted integral field spectrograph such as that selected as one of the firstlight instruments for E-ELT (based on the HARMONI concept, Tecza et al. 2009). The simulations show that the E-ELT spectra will be of sufficient quality to determine the host-galaxy redshift and, crucially, to determine the type of supernova by detecting the presence of the Si II $4000 \AA$ feature (in the observed K band spectrum at redshift of 4). Observations of a sample of 50 SNeIa in the redshift range $1<z<4$ requires 400 E-ELT hours spread over a period of 5 years.

\section{Conclusions}

Two major new facilities are expected to be in operation around the turn of the decade: E-ELT, a general purpose giant telescope, and EUCLID, a survey machine for cosmology and legacy science. Both will operate at optical and near-infrared wavelengths and will be immensely powerful for a wide range of science, including time-domain astronomy. That has been illustrated with the example of observations of distant supernovæ for cosmology, where EUCLID has the potential for NIR imaging of a few thousand SNeIa to intermediate z, and E-ELT will be able to make spectroscopic measurements of SNeIa to $z=4$.

\section{References}

Bode, M., Cruz, M. J., \& Molster, F. J. (Eds.), 2008, The ASTRONET Infrastructure Roadmap, www . astronet-eu.org/IMG/pdf/Astronet-Book.pdf

Freedman, W., et al., 2009, ApJ, 704, 335

Hook I., 2010, in: A. Shearer (ed.), High Time Resolution Astrophysics IV: The Era of Extremely Large Telescopes, Proceedings of Science, 108

Kissler-Patig, M., Kupcu Yoldas, A., \& Liske, J. 2009, ESO Messenger 138, 11

Nobili, S. et al., 2009, ApJ. 700, 1415

Riess, A., et al., 2000, ApJ, 536, 62

Shearer, A., et al.. 2010, arXiv:1008.0605v2

Sullivan, M., et al., 2011, ApJ, 737, 102.

Tecza, M., Thatte, N., Clarke, F., \& Freeman, D., 2009, in: A. Moorwood (ed.), Science with the VLT in the ELT Era, (Netherlands: Springer), p. 267 